\title{
Tracking Indian Tigers using Adhoc Networks \& GIS
}

\author{
Dr. Yesu Babu Adimulam \\ Professor, Dept of CSE \\ CRR Engineering College \\ Eluru \\ Dr.Ramana Banothu \\ Professor, Dept. of CSE \\ WIT, Chevella
}

\author{
G.Charles Babu \\ Professor, Dept of CSE \\ HITS, Bogaram, Hyd
}

\author{
B Sankara Babu \\ Assoc. Prof., Dept of CSE \\ GRIET, Hyd \\ Srinivas Jagirdar \\ Asst. Professor, Dept of CSE \\ Muffakamjah College of Engineering, \\ Hyderabad
}

\begin{abstract}
Indian forests hold over half the world's tiger population. The forest rangers find it very hard to track the tigers. The combination of Wireless technology, Adhoc Networks and GIS can be helpful in this aspect. We propose a system that uses a combination of Adhoc network, GPS \& GIS technologies to help the forest rangers in tracking \& identification of the tigers. The primary focus is on the advantages that Adhoc Networks offer. The Communication between the animal's device and the user can be via GSM cellular network which allows real-time tracking. The communication in forest like remote areas can be established using Ad-hoc networks.
\end{abstract}

Keywords: Adhoc network, GIS, Wireless technology.

\section{INTRODUCTION}

An ad hoc network with their ability to multi hop [1] offers a viable solution for GPS enabled tracking of tigers in Indian forests. Not only does it extend the area that can be covered, but it also provides the ability for nodes to be mobile or nomadic thus accommodating wildlife officers in being able to move network nodes. A GPS-enabled device, such as a GPS Pod(see Figure 1) will normally record and store location data at a predetermined interval or on interrupt by an environmental sensor. These data is relayed to a central data store using an embedded cellular (GPRS), radio. The animal's location can then be plotted against a map in near real-time or, when analyzing the track later, using a GIS package[2].

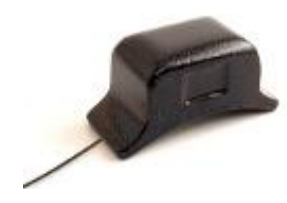

Figure1. GPS Pod

Design constraints should be considered while dealing with the ad hoc networks. Interference in the wireless transmission channel such as topography and vegetation of the area should be dealt with first. The routing algorithm that governs the information flow to the intended station is the second issue. Visualization of the data through a GIS application is the third issue. In this paper all the issues are taken into account when determining the theoretical performance of an ad hoc wireless network for the application of Tiger tracking in the nagarjunsagar forest area using GPS .The rest of this paper will proceed as follows: Section II describes the system architecture and the working of the system. Section III details the simulation $\&$ visualization process. Section IV details the implementation details. The paper is concluded in section V. The future scope is given in section VI.

\section{SYSTEM ARCHITECTURE}

In applications like tiger monitoring, some nodes may have larger battery capacity and more powerful processing capability; which would allow them to serve as data gathering nodes (gateways). Some nodes may only be responsible for sensing and sending collected data to the gateway nodes. Some nodes may be solar powered.

In tracking tigers, four types of nodes are required

1) Animal Node (nomadic) - These individual sensor nodes that are affixed to each of the tigers being tracked.

2) Anchor Nodes (fixed) - These nodes propagate the signals from the animal node to the base station

3) Base Station (fixed) - A data store which has infrastructure to deal with continuous data reception/transmission.

4) Human node (mobile/nomadic)-A PDA or Laptop with a GIS application through which the ranger can track the position of the animal on the move.

The Animal node can be a GPS-POD which is solar powered for limitless GPS data transmission/reception. It is highly programmable easy to mount device. A GPS-POD will normally record and store location data at a pre-determined interval or on interrupt by an environmental sensor. These data is relayed to a central data store or using an embedded cellular (GPRS), radio. The animal's location can then be plotted against a map in near real-time or, when analyzing the track later, using a GIS package or custom software. 


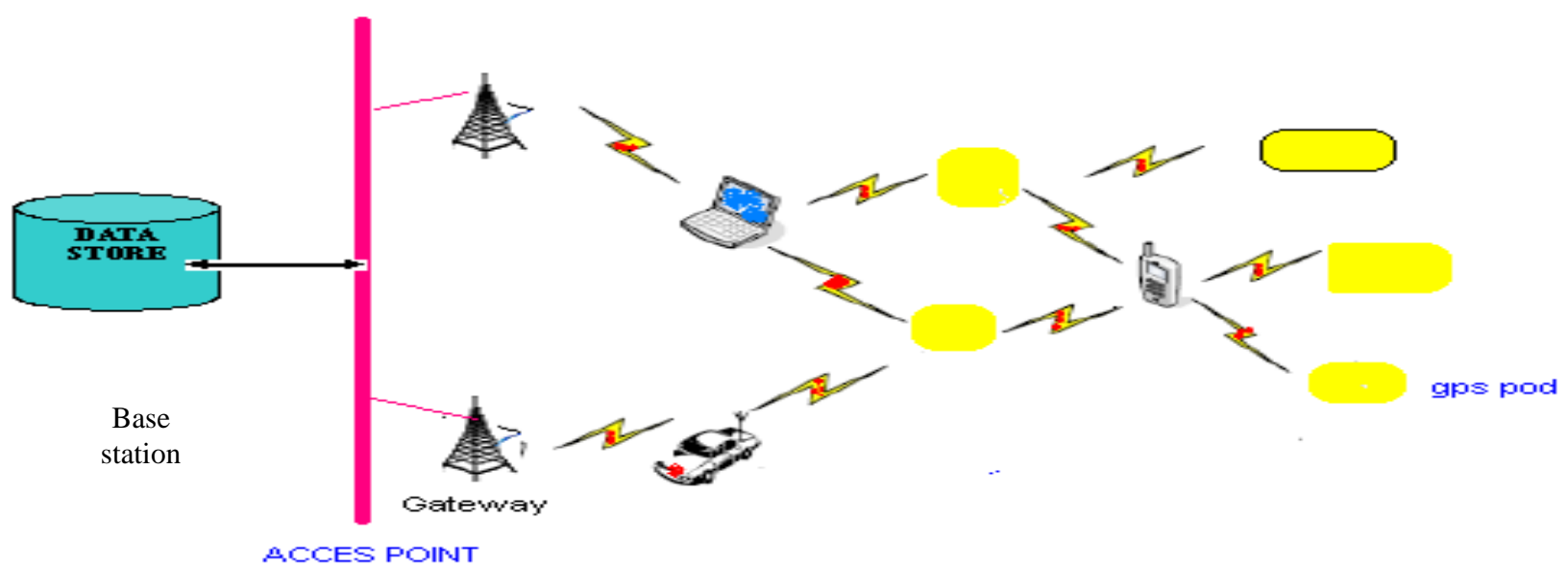

Figure 2. System architecture

There are 2 types of network connections that make the system Adhoc network between the GPS Pods and the ranger PDA's or laptops and then wireless communication using the GPRS between the towers and the node in range. The tower $\&$ the base station consisting of the data store $\&$ some computing devices can have a wireless or wired connection. The access point acts as the authorization point. It screens any unauthorized access to the data store.

\subsection{Working}

The data transmission among mobile nodes in Ad Hoc network adopts the advanced technology of distributed computing. Wireless data transmission by mobile terminals with the Ad-Hoc routing mode is an effective is an effective method to realize wireless GIS. With the help of this technology, it can offer a network linked by many wireless node in short distance, the nodes in large scale can also be linked by multi-hop in nearby nodes to build a large Ad hoc network. In addition, traditional wireless Ad Hoc routing protocols needs to be optimized in application to mobile geospatial information service [2].

First the mobile terminal needs to find the Ad Hoc network gateway to connect with the cellular telecommunication network [3]. The network gateway periodically transmits package all around to broadcast own existence. If the mobile terminal is within one-hop distance from the gateway the, the mobile terminal may find the mobile gateway through the received broadcast information. If the distance is beyond one hop, the mo-bile terminal will send the control package to seek the gateway, and then this mobile terminal will receive from one or more than one reply from the Ad Hoc network gate-way. The mobile terminal will select the gateway with least hop number to connect with the cellular telecommunication network. By using the gateway router, several Ad Hoc networks may be interconnected to enable the mobile node to reach cellular telecommunication network.. In addition, traditional wireless Ad Hoc routing protocols needs to be optimized in application to mobile geospatial information service.

\section{VISUALIZATION THROUGH GIS}

The GIS application (see Figure 3) is developed using oracle maps [4], the latitude longitude of the GPS Pods are continuously transmitted to the data store which is stored using the oracle spatial data base management system. Even the critical information such as the pulse rate can be transmitted to the base station using the GPS Pods. The GIS application is designed to visualize the park. The towers, GPS Pods of the animal $\&$ the base station are marked on the map based on their latitude \& longitude [5]. When the ranger clicks on the GPS Pod symbol its location information, pulse count and personal identification information can be retrieved. The application through its search module (see Figure 3) allows the ranger to search for a particular tiger by entering its name.

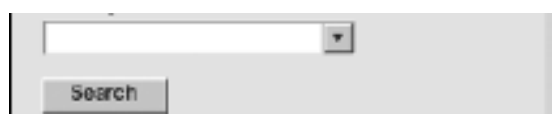

Figure3. Search module

The result of the query (see Figure 4) would give the ranger the exact location of the tiger. He can monitor the pulse rate of the tiger. A sensor attached to the GPS Pod transmits the pulse rate of the tiger either continuously or at regular intervals. The normal pulse rate is between $70-80$ [6] beats per minute. If the pulse rate is zero than it means that the tiger is dead. The ranger can directly rush to the scene and perform his formalities. The current application displays the pulse rate, name \& gender of the tiger through a tool tip. Armed with this information the ranger can continuously track the tigers of the forest. 

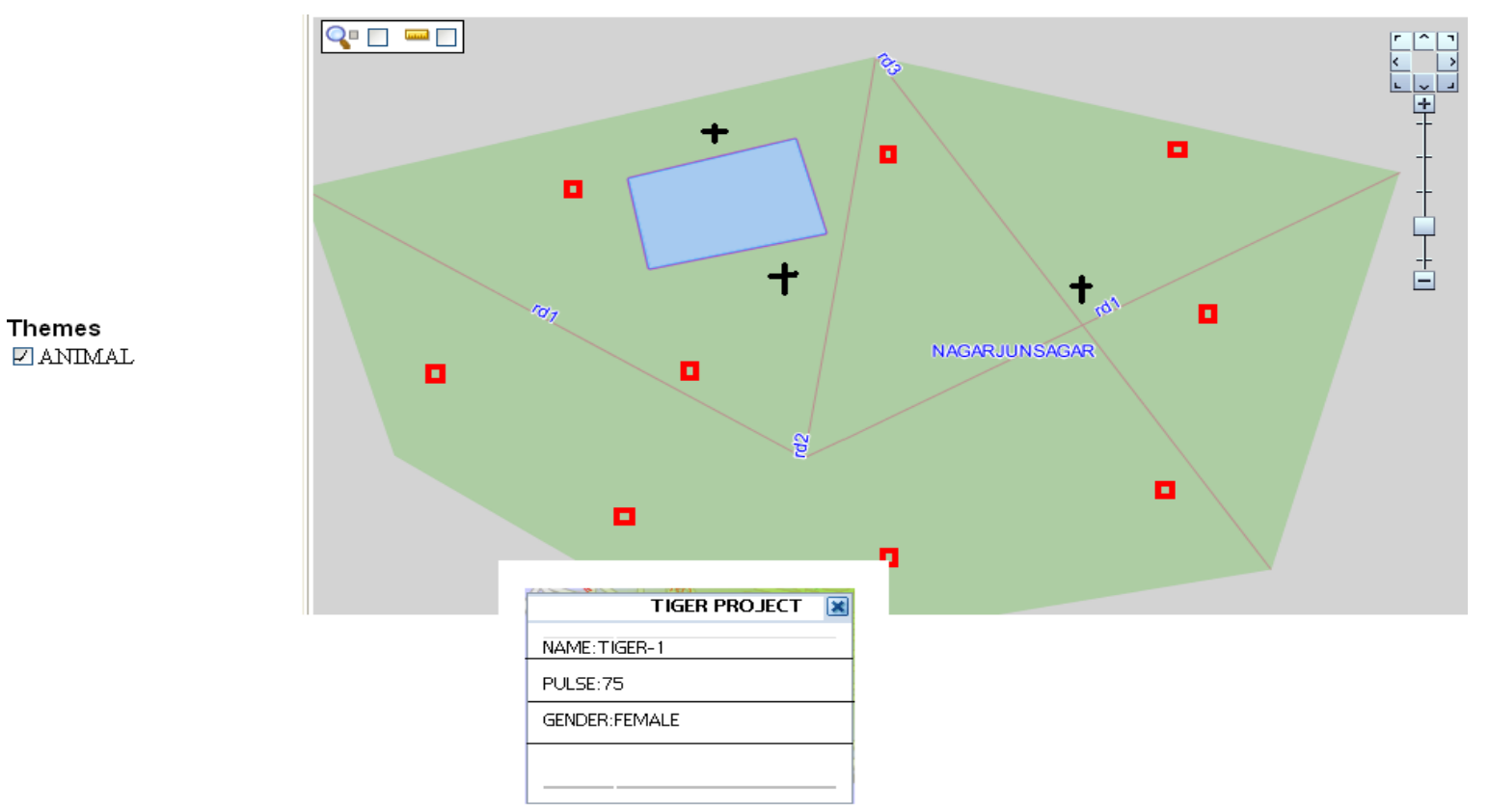

Figure 4. Result of the query

\section{IMPLEMENTATION}

The real world forest like scenario is simulated. We have selected an area of 4.25 square kilometers and visualized it in a map. The GPS Pods are attached to some vehicles on the move. These Pods continuously transmit their current location information as GPS data, which is stored using the oracle spatial data base. The Towers are fixed at a certain location so that they are easy accessible by the GPS Pods. It is found that taking average of the location information of the animal that is being transmitted over a period of time would give us a more accurate position of the tiger. We have used NS-2 to simulate the tests. The system testing was done keeping the same environmental conditions. Therefore to observe the effect of changing environmental conditions, a set of experiments were performed to study the variation in the signal strength due to environmental conditions such as day/night, and vegetation as follows:

1) In Free Space Region on 3rd of October, 2010 at 2 PM

2) In Free Space Region on 3rd of October, 2010 at 9PM

3) Vegetation Region: 4th of October, 2010 at 7:30 PM

The best transmission rate was observed on test conducted in free space region in the morning.

\section{CONCLUSIONS}

Through these tests we observed that the change in environmental condition has a significant effect on the data transmission rate values thus enhancing the error in tracking the location of the tigers. The terrain and the type of flora effect the data transmission. The system is completely dependent on the GPS Pods; hence any physical damage done to the pods will seriously affect the performance of the system.

\section{FUTURE SCOPE}

For the system to work in dense forests more powerful GPS devices are required. A more optimized Adhoc routing protocol is required. The system can be enhanced if even the other animals of the forest are collared as it increases the number of active nodes.

\section{REFERENCES}

[1] Krishna Gorantla, 2006. Routing Protocols in MANETS, .

[2] Fuling Bian, Yun Zhang, 2009 Geospatial Information Service Based On Ad Hoc Network,

[3] Jochen Schiller. 2000 Mobile Communications.

[4] Ravi Kothuri, Albert Godfrind, Euro.2007 Pro Oracle Spatial for Oracle Database $11 \mathrm{~g}$ 